\title{
Miniaturized pulsed laser source for time-domain diffuse optics routes to wearable devices
}

\author{
Laura Di Sieno \\ Jan Nissinen \\ Lauri Hallman \\ Edoardo Martinenghi \\ Davide Contini \\ Antonio Pifferi \\ Juha Kostamovaara \\ Alberto Dalla Mora
}




\title{
Miniaturized pulsed laser source for time-domain diffuse optics routes to wearable devices
}

\author{
Laura Di Sieno, ${ }^{a, *, \dagger}$ Jan Nissinen, ${ }^{b, \dagger}$ Lauri Hallman, ${ }^{b}$ Edoardo Martinenghi, ${ }^{a}$ Davide Contini, ${ }^{a}$ Antonio Pifferi, ${ }^{a, c}$ \\ Juha Kostamovaara, ${ }^{b}$ and Alberto Dalla Mora ${ }^{a}$

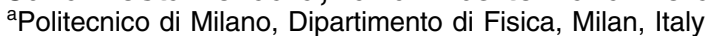 \\ bUniversity of Oulu, Circuits and Systems Research Unit, Oulu, Finland \\ ${ }^{c}$ Consiglio Nazionale delle Ricerche, Istituto di Fotonica e Nanotecnologie, Milano, Italy
}

\begin{abstract}
We validate a miniaturized pulsed laser source for use in time-domain (TD) diffuse optics, following rigorous and shared protocols for performance assessment of this class of devices. This compact source $\left(12 \times 6 \mathrm{~mm}^{2}\right)$ has been previously developed for range finding applications and is able to provide short, high energy ( $\sim 100 \mathrm{ps}, \sim 0.5 \mathrm{~nJ})$ optical pulses at up to $1 \mathrm{MHz}$ repetition rate. Here, we start with a basic level laser characterization with an analysis of suitability of this laser for the diffuse optics application. Then, we present a TD optical system using this source and its performances in both recovering optical properties of tissue-mimicking homogeneous phantoms and in detecting localized absorption perturbations. Finally, as a proof of concept of in vivo application, we demonstrate that the system is able to detect hemodynamic changes occurring in the arm of healthy volunteers during a venous occlusion. Squeezing the laser source in a small footprint removes a key technological bottleneck that has hampered so far the realization of a miniaturized TD diffuse optics system, able to compete with already assessed continuous-wave devices in terms of size and cost, but with wider performance potentialities, as demonstrated by research over the last two decades. () The Authors. Published by SPIE under a Creative Commons Attribution 3.0 Unported License. Distribution or reproduction of this work in whole or in part requires full attribution of the original publication, including its DOI. [DOI: 10.1117/1.JBO.22.8.085004]
\end{abstract}

Keywords: picosecond laser; integrated laser driver; biomedical optics; time-domain diffuse optics; scattering media; time-correlated single-photon counting.

Paper 170207R received Apr. 4, 2017; accepted for publication Jul. 27, 2017; published online Aug. 19, 2017.

\section{Introduction}

Time-domain (TD) diffuse optics was introduced 30 years ago as a promising tool for deep noninvasive probing of the human body and other highly scattering media. ${ }^{1,2}$ The measurement relies on the injection of a picosecond laser pulse in the location of interest (e.g., muscle, brain, or breast); re-emitted light is then collected at a distance on the order of a few centimeters by a time-resolved single-photon detector. The distribution of photon time of flights (DTOF), obtained using time-correlated singlephoton counting (TCSPC) electronics, ${ }^{3}$ encodes information about tissue absorption and scattering, which are related respectively to tissue composition and microstructure. Hence, the technique allows the characterization of tumor lesions ${ }^{4}$ or the detection of brain activation through the change of oxyand deoxyhemoglobin concentrations, ${ }^{5}$ just to mention a few common applications.

Diffuse optics systems have already reached a commercial level with compact and wearable devices, thus being proposed not only for medical applications but also for the consumer market such as the personal monitoring of muscle oxygenation for athletes. However, these devices are always based on continuous-wave $(\mathrm{CW})^{6}$ or frequency-domain ${ }^{7}$ operation, due to the much higher scalability of components operating in such domains. TD systems are potentially quite attractive for the high information content, ${ }^{8}$ in particular for their unique feature of selecting different mean investigated depths using different

\footnotetext{
*Address all correspondence to: Laura Di Sieno, E-mail: laura.disieno@polimi.it
}

†These authors equally contributed to the paper arrival times of detected photons. ${ }^{9,10}$ During the last decade, relevant improvements in terms of cost and complexity have been achieved, ${ }^{11,12}$ squeezing systems from optical tables to rack cabinets, ${ }^{13}$ down to boxes (size: $200 \times 160 \times 50 \mathrm{~mm}^{3}$ ) fitting into a backpack. ${ }^{14}$ However, CW systems are still orders of magnitude smaller and cheaper, thus dominating the market despite their intrinsic limitations; indeed, they suffer from poor information content and high sensitivity to motion artifacts. Additionally, physics itself limits the development of improved CW systems since it has been demonstrated that an ultimate ideal CW system can achieve a significantly lower penetration depth in tissues with respect to an ideal TD one $(\sim 3 \mathrm{~cm}$ instead of $\sim 6 \mathrm{~cm}$ for the same paradigmatic case) and orders of magnitude lower sensitivity to localized heterogeneities. ${ }^{15}$ These ultimate performances for TD are reachable, provided that large area detectors are available in contact with the tissue under investigation (to maximize the collected light) with a proper time-gating mechanism (to reject early-arriving photons), ${ }^{16}$ and that many pulsed sources can be arranged one close to another to maximize the injected light up to the safety limits.

While miniaturized probe-hosted detectors ${ }^{17}$ and also detectors with on-chip timing circuits ${ }^{18}$ have been already well demonstrated in the literature, thus almost reaching the level of compactness required to compete with $\mathrm{CW}$ detection chains, pulsed laser sources still lack the desired level of integration. In a previous work by some of us, ${ }^{15}$ we presented a probe directly hosting a time-gated single-photon detector and a pulsed laser die, thus emitting all the light generated directly into the tissue, without the need of optical fibers. However, while the probe hosted the laser die and its front-end circuitry 
needed to bias it, it still required an external benchtop ultrafast voltage pulse generator and a radio frequency $50 \Omega$ cable to provide electrical pulses to the probe. The transition speed and shape of such pulses are crucial to properly operate the laser diode in gain-switching mode, thus requiring the bulky and expensive radio frequency pulse generator. Apart from the overall dimension and cost, this solution is not acceptable in particular for the design of multichannel systems since it requires an external pulse generator for each laser source of the system. Indeed, the fast electrical pulse cannot be split over different paths without affecting the pulse amplitude and shape, thus impairing the laser output power.

In this work, we take advantage of a technology introduced by some of us in the field of range finding: $:^{19}$ a compact application-specific integrated circuit (ASIC) CMOS driver coupled to a double heterostructure laser diode operated in enhanced gain-switching mode, initially developed for time-of-flight range finding. Despite its low maximum repetition rate $(1 \mathrm{MHz})$ that limits the TCSPC acquisition, diffuse optics can benefit from the high energy $(\sim 0.5 \mathrm{~nJ})$ and narrow $(\sim 100 \mathrm{ps})$ pulses provided. Hence, the goal of this work is to demonstrate the suitability of this technology for TD measurements into highly scattering media, thus removing the last technological barrier preventing the design of wearable TD systems. Also, substantial increase in the laser repetition rate is definitely feasible, provided that the laser driver is redesigned taking into account this requirement that was not addressed in conceiving it for range finding applications.

The paper is organized as follows: Sec. 2 introduces a basic level characterization of the laser (pulse shape, average power, wavelength, and stability over time) with focus on TD diffuse optics requirements. Section 3 deals with validation on tissuemimicking phantoms both in recovering the optical properties of homogeneous media and in detecting a localized absorbing perturbation in a scattering sample. Section 4 reports a proof of concept of in vivo use in monitoring hemodynamic changes occurring in the arm of three volunteers during a venous cuff occlusion. Finally, Sec. 5 presents conclusions and future perspectives.

\section{Basic Characterization of the Laser}

\subsection{Laser Description}

In Refs. 20-23, it has been shown that the energy of short ( $\sim 100$ ps) optical pulse emission in gain-switching mode can be markedly increased using a GaAs/GaAlAs quantum well laser diode with a large ratio of $d_{\text {act }} / \Gamma>2 \mu \mathrm{m}$, where $d_{\text {act }}$ and $\Gamma$ are the active layer thickness and confinement factor, respectively. Here a compact CMOS laser driver fabricated in a 0.35-high-voltage (HV) CMOS technology was used to drive a specially grown quantum well semiconductor laser diode utilizing the enhanced gain-switching mode. The laser diode has a stripe width and length of $7 \mu \mathrm{m}$ and $1.5 \mathrm{~mm}$, respectively, and it generates optical pulses with a peak power of $\sim 0.5 \mathrm{~W}$. The amplitude of the driving current pulse generated by the CMOS laser driver is adjusted with the supply voltage of the driver $\left(V_{\mathrm{HV}}\right)$. The pulsing rate achieved with the CMOS driver is $1 \mathrm{MHz}$. For most of the tests presented in the following, $V_{\mathrm{HV}}$ was set to $18 \mathrm{~V}$, which leads to a current pulse amplitude of $\sim 1.3$ A for about $1 \mathrm{~ns}$. The laser driver (CMOS driver in Fig. 1) is mounted together with the semiconductor laser diode (LD in Fig. 1) and a few other passive discrete components on a small printed circuit board $\left(12 \times 6 \mathrm{~mm}^{2}\right)$ as shown in Fig. 1(a). In addition, the metal can of the laser transmitter and all needed connections except ground, which is connected at the bottom of the board by using vias, are shown in Figs. 1(b) and 1(a), respectively. More details can be found in Ref. 19. To trigger the light emission at the desired rate, we generated a square wave synchronism signal with $5 \mathrm{~V}$ pulses lasting a few tens of nanoseconds at $1-\mathrm{MHz}$ repetition rate.

\subsection{Laser Characterization}

In TD diffuse optics, performances in retrieving optical properties of diffusive media and in localizing deep perturbation can be affected by the instrument response function shape, which is given by the convolution of the laser pulse shape, the detector response, and other jitter contributions. ${ }^{16}$ Its shape is usually evaluated not only in terms of full-width at half maximum (FWHM, i.e., width at 0.5 of the peak value) but also down to some orders of magnitude below the peak value (i.e., 0.1 or 0.01 of the peak value), where the effect of reflections, bumps, or decay tails can enlarge the response, thus affecting performances. A first step of this work is therefore the evaluation of the laser pulse shape at different supply voltages $V_{\mathrm{HV}}$ of the laser, ranging from 17 to $35 \mathrm{~V}$. The setup used was composed of the laser described earlier, whose optical pulses were collected by a $1-\mathrm{mm}$ silica fiber $(\mathrm{NA}=0.39$, Thorlabs $\mathrm{GmbH}$, Germany) that was connected to the input of a U-bracket. Another 1-mm silica fiber exiting from the U-bracket focused the collected photons onto the active area of a photomultiplier (a)

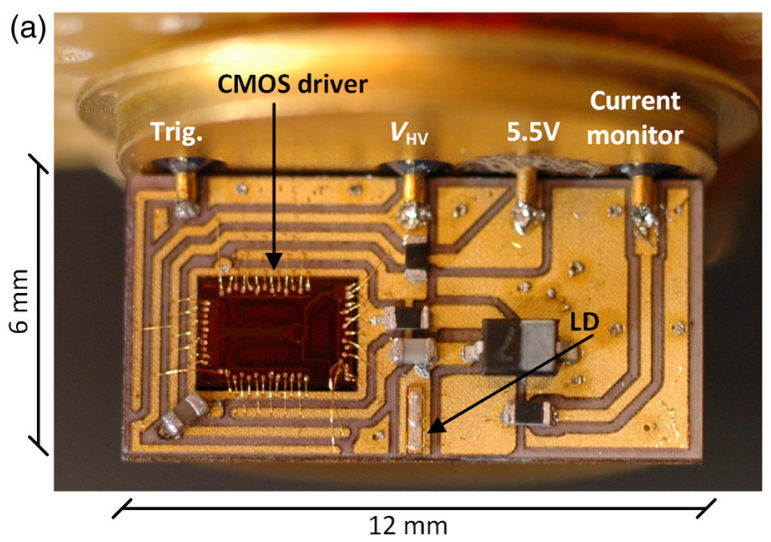

(b)

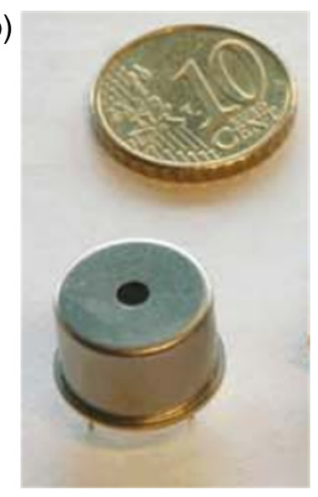

Fig. 1 (a) A photograph of the laser transmitter and (b) a packaged laser transmitter with a coin for scale. 
tube (MCP-PMT with S1 photocathode, Hamamatsu Photonics). Due to a fast temporal response $(<35$ ps FWHM), free from slow tails, this detector guarantees the reconstruction of the generated optical pulse with negligible distortions over a wide dynamic range ( $\sim 4$ orders of magnitude). To adjust the power injected in the collection fiber, we used a variable optical attenuator (VOA) (NDC-100C-4, Thorlabs GmbH, Germany) that was inserted in the clear path of the U-bracket. The "start" signal to the TCSPC board (SPC-130, Becker\&Hickl GmbH, Germany) was fed by the PMT whereas the "stop" signal was provided by the main synchronism. For each voltage, we recorded the DTOF for 60 repetitions of $1 \mathrm{~s}$ each.

In Fig. 2(a), some of the recorded output pulse shapes for $V_{\mathrm{HV}}=18,22,26,30$, and $34 \mathrm{~V}$ are reported, corresponding with current pulse amplitudes of $1.27,1.53,1.76,2.00$, and 2.22 A, respectively.

It is possible to notice that the higher the voltage (i.e., the larger the current pulse amplitude), the steeper the rising edge of the emitted laser pulse, and the wider the temporal shape of the laser.

The trend is more marked at voltages lower than $25 \mathrm{~V}$. Indeed, looking at Fig. 2(b) (right axis), the center of gravity (CoG, i.e., the first moment) of the recorded curves, computed at different thresholds $(0.5,0.1$, and 0.01 of the peak amplitude), after a $V_{\mathrm{HV}}$ of about $25 \mathrm{~V}$ reaches an almost constant value (maximum deviation $<2 \%$ ), if data at 27 and $28 \mathrm{~V}$ are not taken into account. Indeed, for those $V_{\mathrm{HV}}$, an unexpected behavior, not in line with the overall trend is noticed and in such points a maximum deviation from average value (computed in all other points of the stable region, $V_{\mathrm{HV}}>2.5 \mathrm{~V}$ ) is $4.5 \%$. The abnormal discontinuity might be caused by the relaxation oscillation of the semiconductor laser diode, which is a normal behavior when working in the gain-switching mode. At these specific supply voltages, the second peak of the relaxation oscillation has exceeded a certain level, thus increasing the pulse width, and then suddenly dropped because of an electrical disturbance of the driving current of the laser diode. This behavior can be avoided with better transmitter circuit board design.

The variation of the CoG position upon increasing the bias voltage highlights whether the waveform dynamic range is dominated by a smooth rise of the power with time or by decay tails or bumps after the peak power. In particular, from Fig. 2(b), we can see that at $V_{\mathrm{HV}}<20 \mathrm{~V}$ the position of the $\mathrm{CoG}$ is almost independent of the considered threshold, in agreement with the fact that the pulse shape is quite symmetric in the first two-orders of magnitude from the peak. On the contrary, at $V_{\mathrm{HV}}>20 \mathrm{~V}$, the CoG computed at the threshold of 0.5 is $\sim 100$ ps lower than those computed at lower thresholds. This is due to the fact that the pulse shape has a steeper rise on the left and a higher tail on the right, which affects the CoG dependence with the threshold.

Upon an increase in the applied voltage, an increase of the width at level 0.5 (i.e., the FWHM) of the temporal curve can be noticed (left axis). Indeed, the FWHM is 122 ps at $V_{\mathrm{HV}}=17 \mathrm{~V}$ while it reaches $253 \mathrm{ps}$ at $V_{\mathrm{HV}}=35 \mathrm{~V}$. In all cases, the FWHM is lower than 260 ps. These performances are compatible with TD diffuse optics measurements, where lasers with FWHM of about 100 to $200 \mathrm{ps}$ are typically used. ${ }^{11}$ Additionally, laser pulses of a few hundred picoseconds width at 0.01 of the peak value have been already demonstrated suitable for the diffuse optics application in a previous work. ${ }^{16}$ The $V_{\mathrm{HV}}$-dependent shift in the $\mathrm{CoG}$ of the laser shape is not a problem for diffuse optics applications, where the temporal reference is given by the acquisition of the instrument response function (see Ref. 24).

We also analyzed the emission spectrum of the laser as a function of $V_{\mathrm{HV}}$ by means of a spectrometer (OceanOptics, NIRQuest series), as shown in Fig. 2(c). The mean central wavelength is about $797 \mathrm{~nm}$. In Fig. 2(d), both the width of the emission spectrum at different levels from the peak wavelength and the centers of gravity as a function of $V_{\mathrm{HV}}$ are shown. The spectral width increases upon higher $V_{\mathrm{HV}}$, while for the center of gravity there is no clear trend. Indeed, in the range of $V_{\mathrm{HV}}$ between 17 and $20 \mathrm{~V}$, the CoG moves toward shorter wavelengths for increasing $V_{\mathrm{HV}}$, while for $V_{\mathrm{HV}}>20 \mathrm{~V}$ it shifts toward longer wavelengths. The spectral width (FWHM) of the laser [Fig. 2(d), left axis) is always lower than $8 \mathrm{~nm}$. This value is very close to the one of the state-of-the-art TD spectroscopy setup ${ }^{25}$ that features a spectral bandwidth of $7 \mathrm{~nm}$ at $1350 \mathrm{~nm}$. For this reason, the proposed laser can be considered suitable also for spectroscopy measurements.

Using a power meter (Thorlabs $\mathrm{GmbH}$, calibration: $800 \mathrm{~nm}$ ), we measured the average power emitted from the laser at the different $V_{\mathrm{HV}}$ values. Results are shown in Fig. 2(e). The measured power has a clear linear behavior, as underlined by the linear fit (black dotted line) that approximates with high accuracy the experimental data $\left(R^{2}>0.999\right)$. Due to heat dissipation issues of the driver chip, it was not possible to explore the laser power behavior for $V_{\mathrm{HV}}>34 \mathrm{~V}$. Even at this low repetition rate $(1 \mathrm{MHz})$, the average power is suitable for diffuse optical measurements, being of the same order of magnitude of the output power of state-of-the-art fiber-based TD clinical systems. ${ }^{26}$ The measured power consumption at $1 \mathrm{MHz}$ and at $V_{\mathrm{HV}}=18 \mathrm{~V}$ is $50 \mathrm{~mW}\left(160 \mathrm{~mW} @ V_{\mathrm{HV}}=34 \mathrm{~V}\right)$, thus making this source also compatible with battery operation.

Finally, we tested the output power stability of the laser system following the standardized procedures defined by the basic instrumental performances protocol. ${ }^{24}$ The working point was set at $V_{\mathrm{HV}}=18 \mathrm{~V}$, where the laser pulse features an FWHM of $100 \mathrm{ps}$ and an average output power of $62 \mu \mathrm{W}$. Even if the emitted power is only $12 \%$ of the maximum value obtained at $V_{\mathrm{HV}}=34 \mathrm{~V}$, the narrow pulse (99 ps FWHM) should ensure better performance in recovering optical properties of highly scattering media. In this case, we decided to use an SiPM module as a detector ( $1 \mathrm{~mm}^{2}$ active area, see Ref. 27 for details), which is insensitive to thermal perturbations, thus ensuring the possibility to properly monitor possible fluctuations of the laser optical power. Hence, photons exiting from the laser were coupled using a collimator into a silica fiber (core diameter: $1 \mathrm{~mm}$ ); a VOA hosted in a U-bracket was used to properly attenuate the power to fit the single-photon statistics, ${ }^{3}$ attenuated light was collected by another 1-mm-core silica fiber (whose propagation modes were completely filled using a thin layer of Teflon) coupled to the SiPM module due to a doublets of lens performing 1:1 imaging $(f=30 \mathrm{~mm})$. We recorded the DTOF for about 100 min with an integration period of $10 \mathrm{~s}$.

For each DTOF, the background noise was subtracted and then both the overall number of counts (i.e., area of the curve) and the moments (at 0.5, 0.1, and 0.01 levels) were computed. Results are shown in Fig. 2(f). After an initial warm-up of $20 \mathrm{~min}$, the number of counts and the center of gravity are almost stable (within $\pm 1 \%$ and \pm 10 ps, respectively), apart from fast changes possibly related to instabilities that could be improved by employing proper heat dissipation strategies. 


\section{(a)}

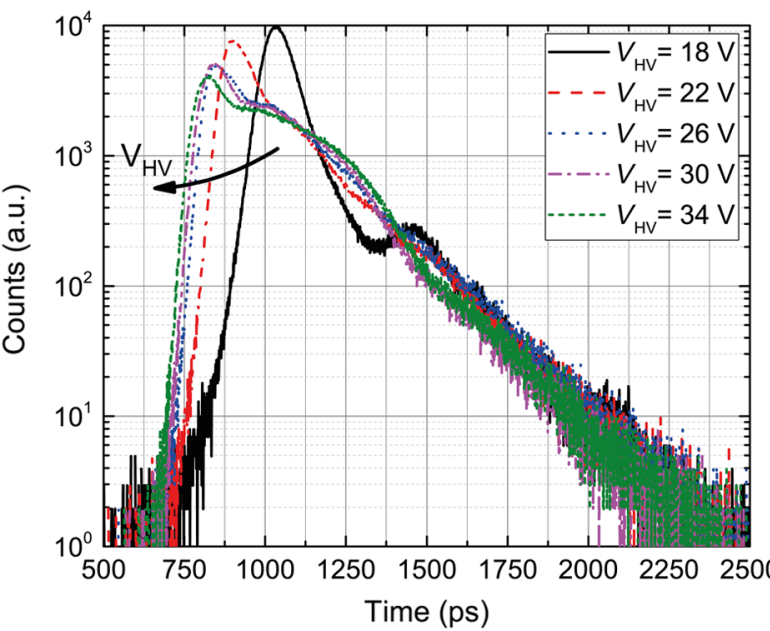

(c)
ह
की
के

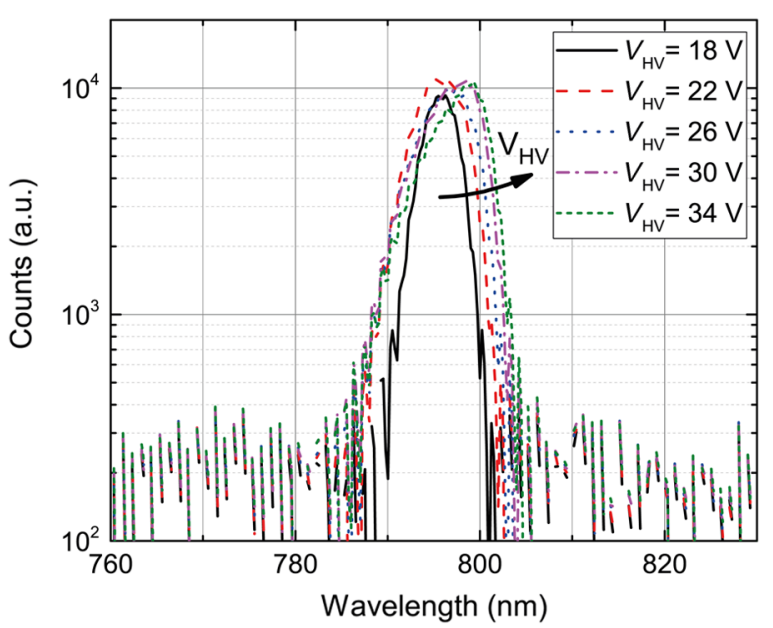

(e)

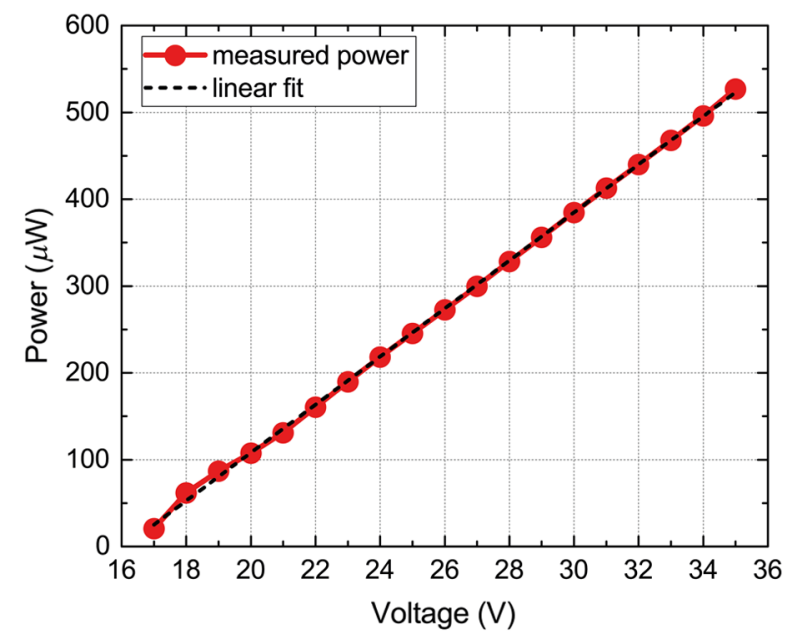

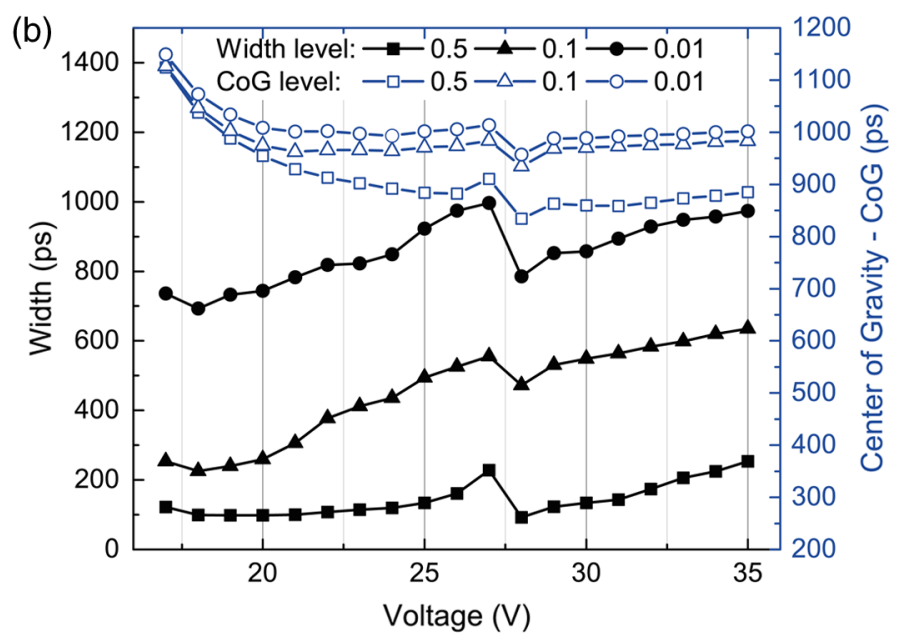

(d)
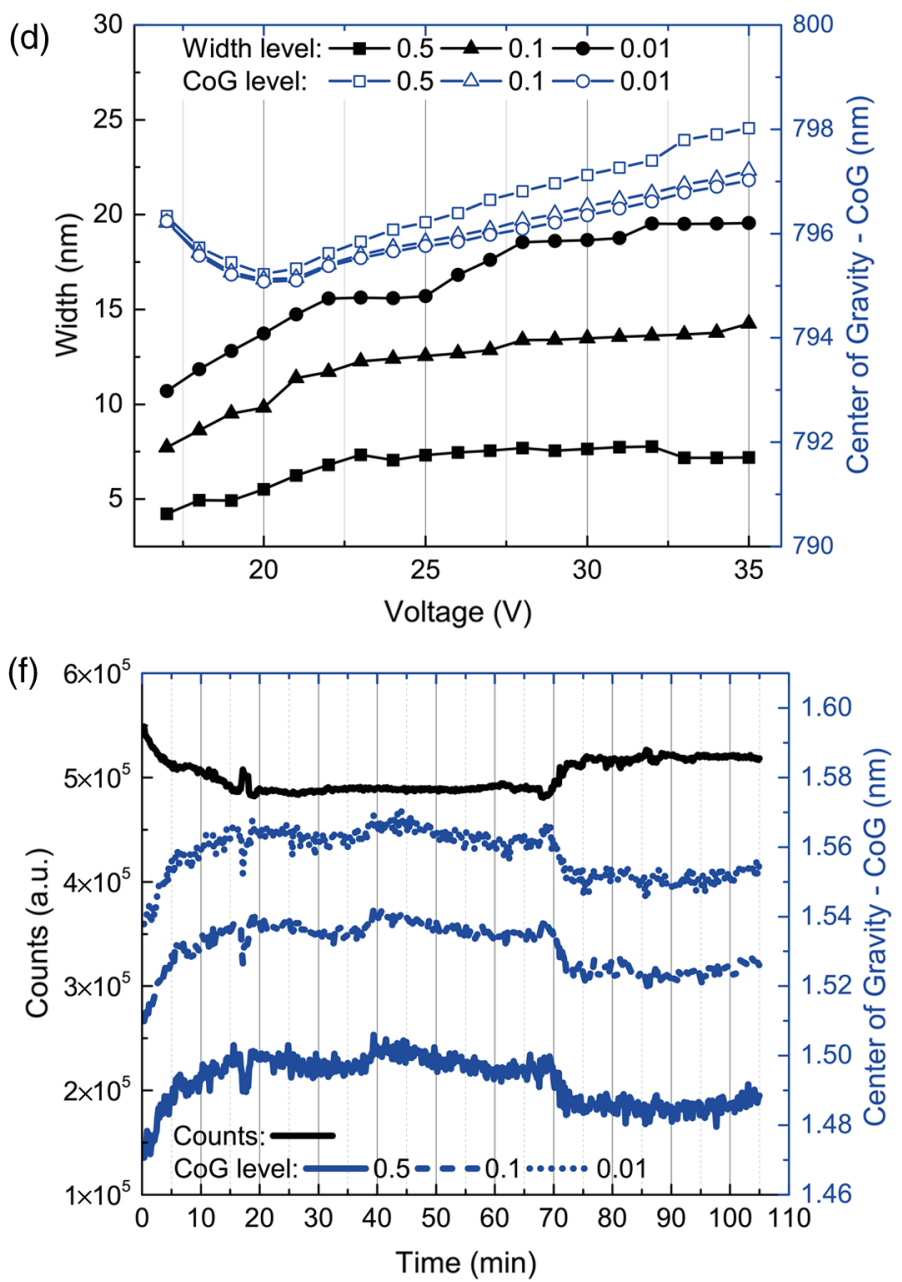

Fig. 2 Characterization of the laser: (a) optical pulse shapes at different $V_{\text {HV }}$ values, (b) temporal characteristics, (c) emission spectrum, (d) emission spectrum characteristics, (e) emitted average power at different $V_{\mathrm{HV}}$, and (f) optical pulse stability over time.

\section{Validation on Phantom}

With the aim of using a technology that already allows the fabrication of wearable devices, for the phantom measurements we decided to use the probe-hosted SiPM detector reported in Ref. 17, thus also maximizing the collection of diffused light from the phantom due to the large numerical aperture of the
SiPM and also avoiding the use of both optical fibers or lenses. While the detector was placed directly in contact with the phantom, the laser was inserted into a metallic case (with a hole drilled for laser output) to avoid electromagnetic interferences between the pulsed laser current and the electrical output of the detector, which can introduce distortions in the DTOF. A 
VOA was inserted between the laser and the sample surface to adjust the injected power in order not to exceed the maximum count rate allowed by the TCSPC technique to avoid pile-up distortions (i.e., a count rate of a few percent of the laser pulse rate of $1 \mathrm{MHz}$ ). $V_{\mathrm{HV}}$ was set to $18 \mathrm{~V}$.

\subsection{MEDPHOT Protocol}

To assess the suitability of the proposed compact setup in retrieving the optical properties - absorption $\left(\mu_{\mathrm{a}}\right)$ and reduced scattering coefficient $\left(\mu_{\mathrm{s}}^{\prime}\right)$ - of homogeneous phantoms, we adopted the linearity test defined by the MEDPHOT protocol. ${ }^{28}$ The linearity test consists of the measurement of four series of solid phantoms featuring different reduced scattering coefficients (labeled A, B, C, and D with $\mu_{\mathrm{s}}^{\prime}=2.8,5.6,8.5$, and $11.27 \mathrm{~cm}^{-1}$ at $800 \mathrm{~nm}$, respectively). Each series is composed of eight phantoms with constant $\mu_{\mathrm{s}}^{\prime}$ and covering a wide range of absorption coefficients (labeled from 1 to 8 and spanning $\mu_{\mathrm{a}}$ from 0 to $0.32 \mathrm{~cm}^{-1}$ at step of $\sim 0.05 \mathrm{~cm}^{-1}$ at $800 \mathrm{~nm}){ }^{28}$ Despite the low repetition rate, the high laser power permitted measuring all phantoms in transmittance geometry (thickness of the phantom around $45 \mathrm{~mm}$ ) and we collected the DTOF with 60 repetitions of $1 \mathrm{~s}$ each.

To obtain the values of $\mu_{\mathrm{a}}$ and $\mu_{\mathrm{s}}^{\prime}$, the measured DTOFs were fitted using an analytical solution of the radiative transport equation under the diffusion approximation, ${ }^{29}$ convoluted with the instrument response function (previously measured, data not shown).
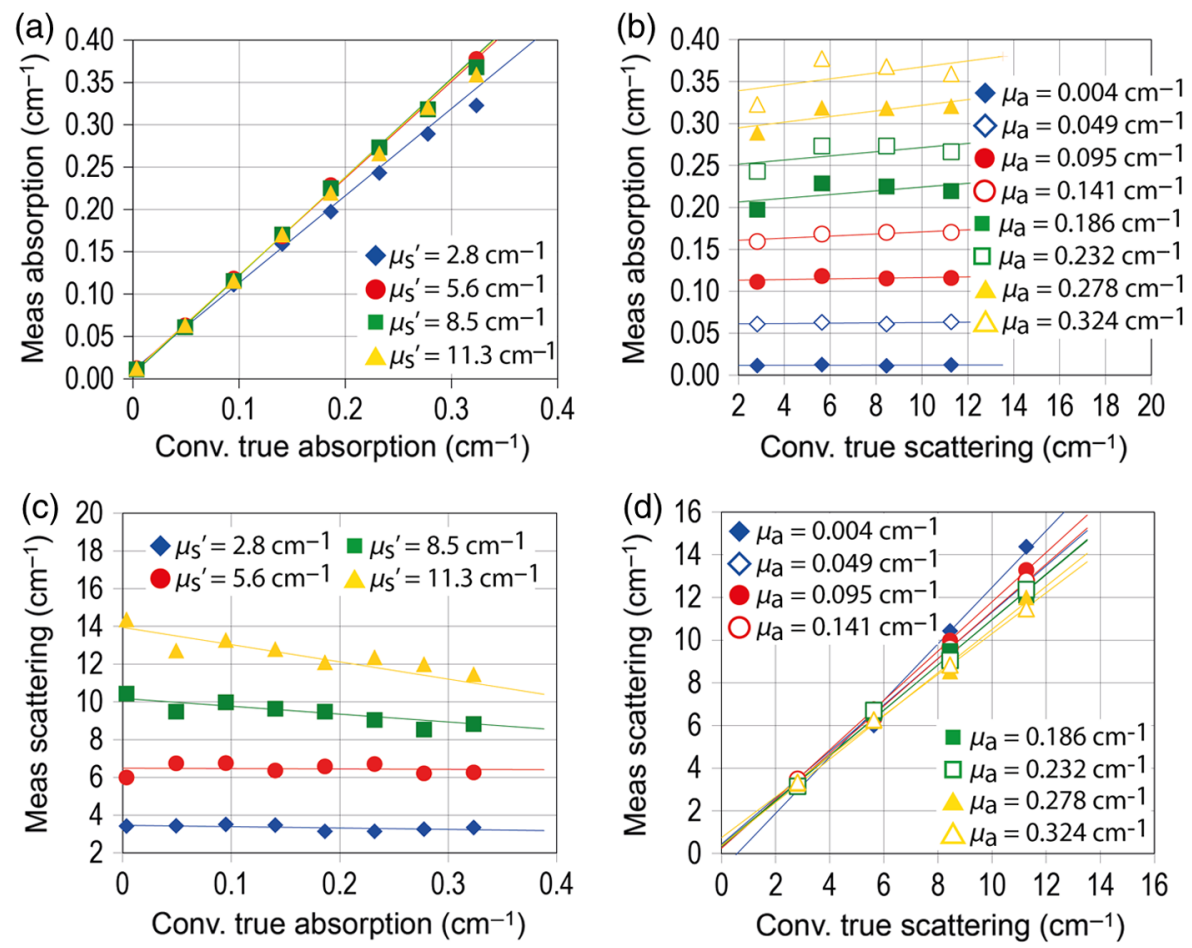

Geometry used: transmittance Sample thickness: $45 \mathrm{~mm}$

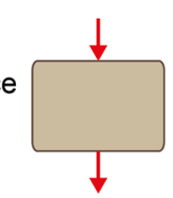

Fig. 3 Results from MEDPHOT protocol. (a) Displays absorption linearity, (b) the coupling of the absorption coefficient to the scattering one, (c) the scattering linearity, (d) the coupling of the scattering coefficient to the absorption one. For all measurements the laser driver was operating at $V_{\mathrm{HV}}$ of $18 \mathrm{~V}$. 
background. For this purpose, we used the "contrast" (C) and "contrast-to-noise ratio" (CNR) tests defined in the "nEUROPt protocol." $"$ For its implementation, we used a switchable solid phantom (described in Ref. 31) that allows one to move an absorbing perturbation along one direction. The optical properties of the background phantom were $\mu_{\mathrm{a}}=0.1 \mathrm{~cm}^{-1}$ and $\mu_{\mathrm{s}}^{\prime}=$ $8 \mathrm{~cm}^{-1}$ while the perturbation features a $\Delta \mu_{\mathrm{a}}$ of $0.17 \mathrm{~cm}^{-1}$ in a volume of $1 \mathrm{~cm}^{3}$.

Measurements were done in reflectance geometry (sourcedetector distance: $3 \mathrm{~cm}$ ) with the inclusion in the center between the laser and the detector. The perturbation was moved in depth, from 5 to $35 \mathrm{~mm}$ (steps of $5 \mathrm{~mm}$ ) with respect to the surface of the phantom. We computed contrast and CNR in time windows (i.e., subdividing the whole DTOF curve into portions) as defined in the nEUROPt protocol. ${ }^{30}$ In particular, the contrast was calculated summing up all the counts acquired within selected time windows $\left(t_{w}\right)$ of the DTOFs for each position of the inclusion $(z)$ using

$\mathrm{C}\left(t_{W,}, z\right)=\frac{N_{0}\left(t_{W}, z\right)-N\left(t_{W}, z\right)}{N_{0}\left(t_{W}, z\right)}$,

in which $N_{0}$ is the number of photons collected in the unperturbed case (inclusion far-i.e., deeper than $7 \mathrm{~cm}$-from source and detector, thus its effect is completely negligible), while $N$ is the number of photons collected with the perturbation (i.e., inhomogeneous medium). Each time window was 500 ps wide and their temporal positions (i.e., opening of the time window) were referred to as the peak of the IRF. This calculation was performed for all depths $z$ of the inclusion.

For CNR, we made use of the definition given in Ref. 30

$\mathrm{CNR}=\frac{\Delta N\left(t_{w}\right)}{\sigma\left[N_{0}\left(t_{w}\right)\right]}=\frac{N_{0}\left(t_{w}\right)-N\left(t_{w}\right)}{\sigma\left[N_{0}\left(t_{w}\right)\right]}$, where $\Delta N$ was taken over the 60 repetitions for the time window under consideration. The denominator $\sigma\left[N_{0}\left(t_{w}\right)\right]$ was computed as the standard deviation (over the 60 repetitions) of the counts summed in the homogeneous acquisitions. Figures 4(a) and 4(b) show the graphs of contrast and CNR as a function of depth, respectively, computed at different delays of the time window.

As expected: (i) the contrast decreases upon increasing depth of the inclusion due to the broadening of the sensitivity profile and (ii) deeper inclusions $(z>5 \mathrm{~mm})$ are more visible at longer delays of the time window ( $>500 \mathrm{ps}$ ) due to the longer pathlength of late-arrival photons.

Considering a $\mathrm{CNR}=1$ as a threshold for detectability of a perturbation, the perturbation can be detected at depths up to $25 \mathrm{~mm}$ (with time window starting at $2000 \mathrm{ps}$ : $C=0.03$ and $\mathrm{CNR}=1.77$ in Fig. 3). Notwithstanding the compactness of both the laser system and the detector, these values are comparable to other bulkier state-of-the-art systems. ${ }^{13}$

\section{Validation In Vivo}

As a last proof of concept of the proposed laser device, we used the same setup described for the protocols on phantom to perform in vivo measurements of venous occlusions on healthy volunteers. The protocol was approved by the ethical committee of Politecnico di Milano and was conducted in compliance with the declaration of Helsinki. All volunteers gave their written informed consent to participate.

We varied the separation between source and detector to have a level of signal on the detector down to the single-photon level. In this way, the count rate was about 120,35 , and $150 \mathrm{kcps}$ at a source-detector distance of $5.5,4.5$, and $5 \mathrm{~cm}$, respectively, for subjects 1,2 , and 3 . The minimum separation between injection and detection points was $4.5 \mathrm{~cm}$ because the dimension of the

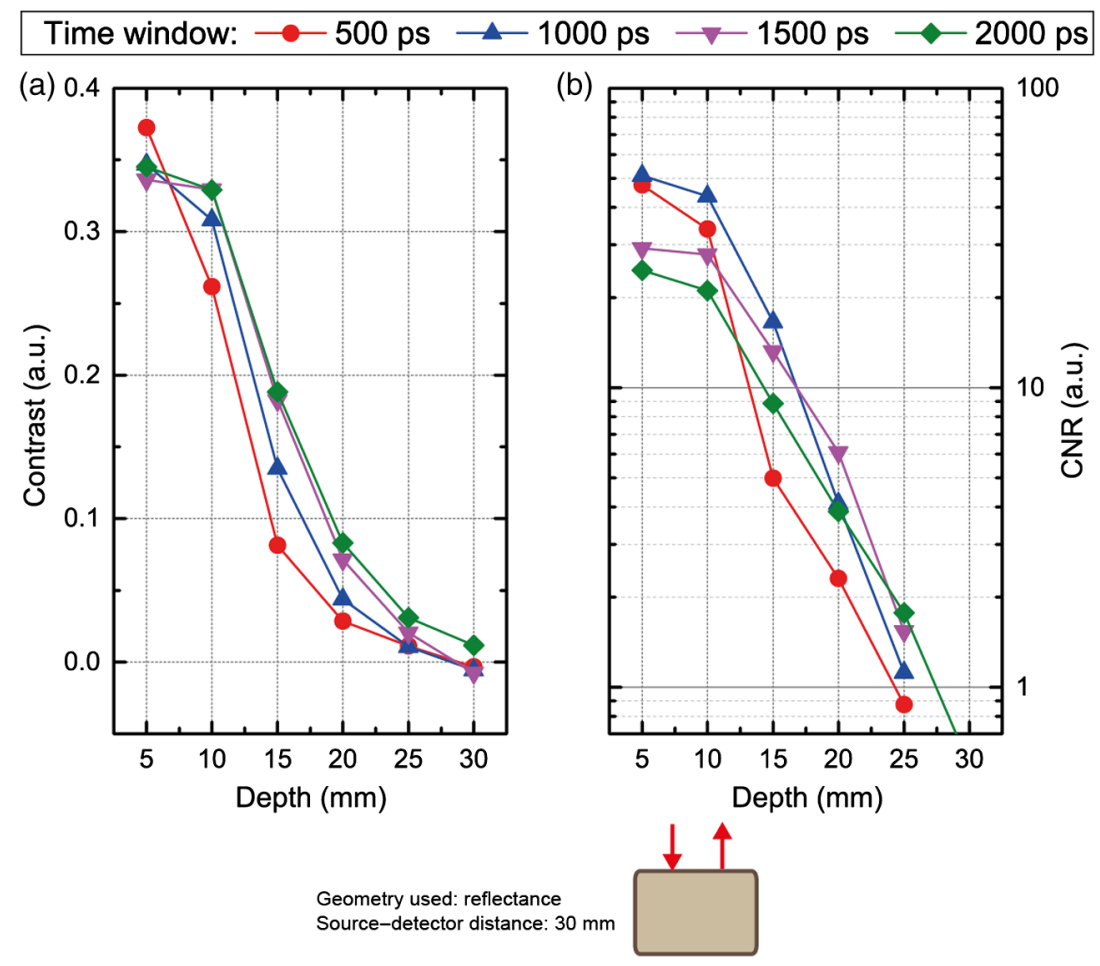

Fig. 4 (a) Contrast and (b) CNR computed for different temporal windows with a perturbation $\Delta \mu_{\mathrm{a}}$ of $0.17 \mathrm{~cm}^{-1}$ in a volume of $1 \mathrm{~cm}^{3}$. 
(a)

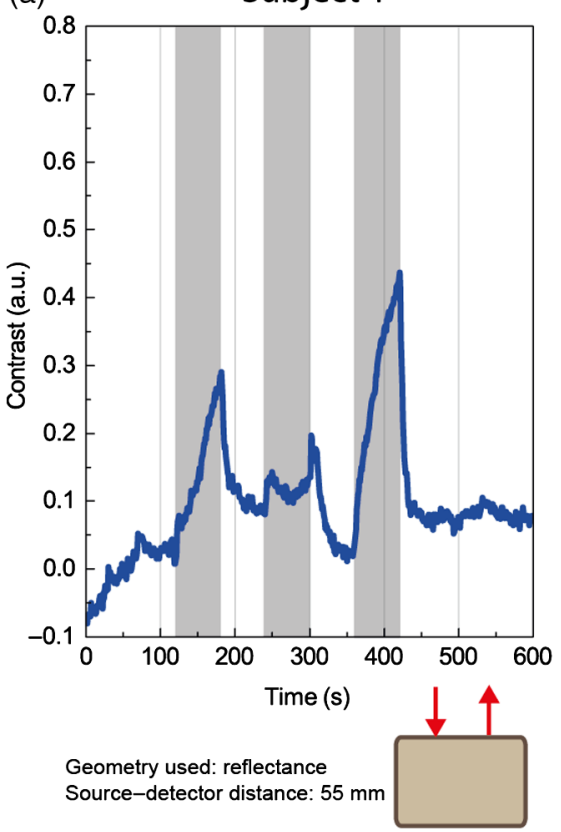

(b)

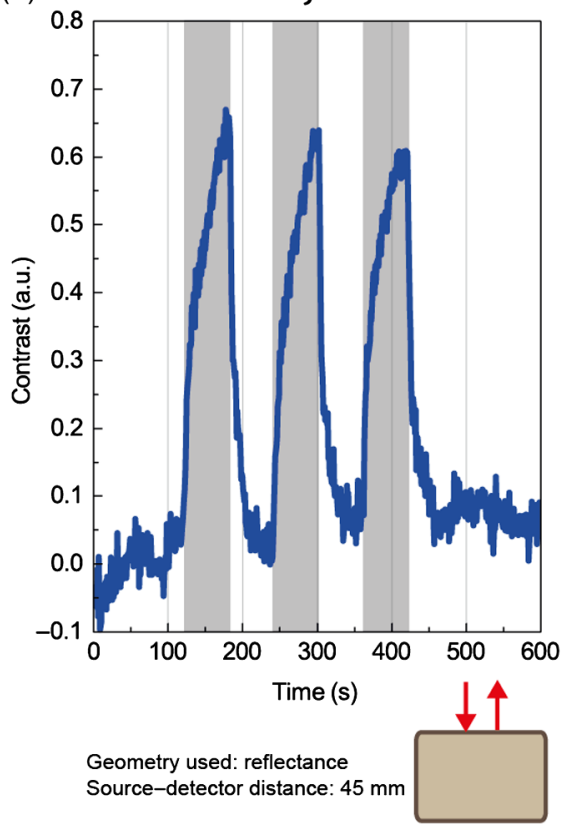

(c)

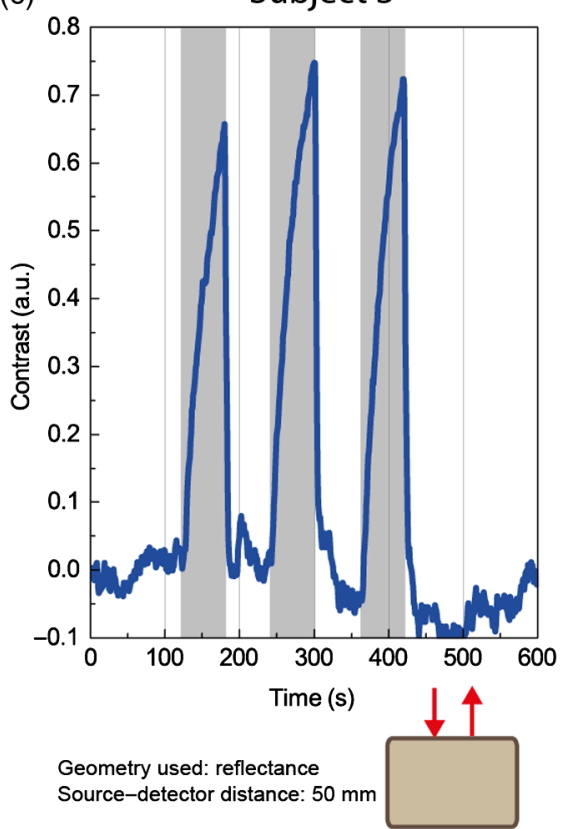

Fig. 5 Results for three different subjects for the venous occlusion measurements.

cases hosting the source and detector, which were mounted on two different printed circuit boards.

The occlusion was induced by a sphygmomanometer cuff placed on the subject's forearm. The measurement protocol was made of three subsequent occlusions, more precisely we had: $120 \mathrm{~s}$ of baseline (i.e., no obstruction of blood flow in the arm) followed by a block repeated three times consisting of $60 \mathrm{~s}$ of occlusion followed by $60 \mathrm{~s}$ of recovery, and then a final recovery phase of $120 \mathrm{~s}$. To induce a venous occlusion (i.e., complete block of venous blood circulation), the pressure applied to the cuff had a value between the own diastolic and systolic ones of each subject measured before starting the experiment.

We made use of the definition given in Eq. (1) to compute contrast, where $N_{0}$ is the mean number of counts acquired during the first $120 \mathrm{~s}$ of rest state. The time window used for analysis is $500 \mathrm{ps}$ wide and started $2.6 \mathrm{~ns}$ after the peak of the IRF. For this experiment, we decided to adopt the time-gated analysis (see Ref. 32) to increase the signal-to-noise ratio with respect to the fitting procedure, which would have instead lead to the recovery of absorption and reduced scattering coefficients.

Figure 5 shows the contrasts obtained for the three subjects. In almost all cases, there is a clear task-related trend, with an increase of contrast (i.e., a decrease of counts) during the phases in which the occlusion was performed (gray rectangles in the figure) meaning an increase in absorption. Then, when the occlusion was removed, the contrast went back close to baseline values, as expected.

Considering the central wavelength of the laser of $796 \mathrm{~nm}$, which is close to the isosbestic point of oxy- and deoxyhemoglobin (located at $797 \mathrm{~nm}$ ), the system is sensible to the overall concentration of hemoglobin, thus the increase in contrast is in agreement with the expected increase in the blood volume due to the venous occlusion. Indeed, during the occlusion, the deoxyhemoglobin cannot be washed out, while the arterial blood flow is not altered. For subject 1, results are less clear, possibly due to a suboptimally working occlusion.

\section{Conclusions}

In this paper, we evaluated the performance of a compact laser device based on the combination of a CMOS ASIC driver coupled to a heterostructure laser diode operated in enhanced gain-switching mode in the TD diffuse optics application. Originally, this laser transmitter was developed for time-offlight range finding applications. Due to its miniaturized size, this technology is potentially able to allow the fabrication of pulsed sources with the same compactness of continuouswave sources employed in currently commercially available diffuse optics systems, but allowing much better performances due to inherent advantages of measurements performed in the TD, such as (i) possibility to disentangle absorption from scattering when measuring optical properties of highly scattering media, (ii) reduced sensitivity to motion artifacts, (iii) higher penetration depth in the tissue, and (iv) possibility to disentangle optical properties of different layers of the measured tissue due to the unique feature of the TD to encode the information of different depths to different arrival delays of photons with respect to the injection time. Additionally, the low power consumption of 50 to $160 \mathrm{~mW}$ (depending on the operative conditions) makes this component compatible with battery operation.

To offer a wide overview of the performances, we characterized the laser system at different levels, starting from its basic performances (optical pulse shape, emission spectrum, and average optical power). Additionally, we combined the source with one of the most recent developments of a compact time-resolved detector based on SiPM technology, which can be easily hosted directly in the probe in contact with the tissue under investigation due its small footprint. The resulting TD diffuse optics system was characterized on phantoms following rigorous protocols for performance assessment of this kind of a system both in recovering the optical properties of homogeneous diffusive media and in detecting localized absorption perturbations. The obtained performances are comparable with state-ofthe-art systems that make use of extremely more bulky and 
expensive lasers and detectors. As a final validation, we tested the system in vivo during a venous cuff occlusion applied on the forearm of three healthy volunteers. Again, the system performance in detecting signal changes due to variations of the total blood concentration during the occlusion is in line with state-ofthe-art diffuse optics systems based on previous generation notscalable technologies. ${ }^{26}$

The results reported here could represent the overcoming of the last bottleneck preventing the design of compact, wearable, and inexpensive TD diffuse optical devices. Together with other advances in photonics components, such as fully integrated TCSPC detection chains, ${ }^{18}$ and probe-hosted solid-state detectors, it is expected within the next few years the development of miniaturized fully operative high-performance TD systems, opening new market scenarios for medical devices but also for consumer appliances.

\section{Disclosures}

No conflicts of interest, financial or otherwise, are declared by the authors.

\section{Acknowledgments}

This project has received funding from the European Union's Horizon 2020 research and innovation programme under grant agreement no. 654148 Laserlab-Europe, the Academy of Finland (Centre of Excellence in Laser Scanning Research, contract nos. 272196, 255359, 283075, and 251571) and the Finnish Funding Agency for Innovation (TEKES).

\section{References}

1. D. T. Delpy et al., "Estimation of optical pathlength through tissue from direct time of flight measurement," Phys. Med. Biol. 33(12), 1433-1442 (1988).

2. B. Chance et al., "Comparison of time-resolved and -unresolved measurements of deoxyhemoglobin in brain," Proc. Natl. Acad. Sci. U. S. A. 85(14), 4971-4975 (1988).

3. W. Becker, Advanced Time-Correlated Single-Photon Counting, Springer, Berlin (2005).

4. P. Taroni et al., "Noninvasive assessment of breast cancer risk using time-resolved diffuse optical spectroscopy," J. Biomed. Opt. 15(6), 060501 (2010).

5. L. Di Sieno et al., "Functional near-infrared spectroscopy at small source-detector distance by means of high dynamic-range fast-gated SPAD acquisitions: first in-vivo measurements," Proc SPIE 8804, 880402 (2013).

6. D. T. Delpy and M. Cope, "Quantification in tissue near-infrared spectroscopy," Philos. Trans. R. Soc. B: Biol. Sci. 352(1354), 649-659 (1997).

7. T. Durduran et al., "Bulk optical properties of healthy female breast tissue," Phys. Med. Biol. 47(16), 2847-2861 (2002).

8. T. Durduran et al., "Diffuse optics for tissue monitoring and tomography," Rep. Prog. Phys. 73(7), 076701 (2010).

9. J. Steinbrink et al., "Determining changes in NIR absorption using a layered model of the human head," Phys. Med. Biol. 46(3), 879-896 (2001).

10. F. Martelli et al., "There's plenty of light at the bottom: statistics of photon penetration depth in random media," Sci. Rep. 6, 27057 (2016).

11. A. Pifferi et al., "New frontiers in time-domain diffuse optics, a review," J. Biomed. Opt. 21(9), 091310 (2016).

12. L. Di Sieno et al., "Diffuse optics using a dual window fast-gated counter," Appl. Opt. 53(31), 7394-7401 (2014).

13. A. Torricelli et al., "Time domain functional NIRS imaging for human brain mapping," Neurolmage 85(1), 28-50 (2014).

14. M. Buttafava et al., "A compact two-wavelength time-domain NIRS system based on SiPM and pulsed diode lasers," IEEE Photonics J. 9(1), 7800114 (2017).
15. A. Dalla Mora et al., "Towards next-generation time-domain diffuse optics for extreme depth penetration and sensitivity," Biomed. Opt. Express 6(5), 1749-1760 (2015).

16. D. Contini et al, "Effects of time-gated detection in diffuse optical imaging at short source-detector separation," J. Phys. D: Appl. Phys. 48(4), 045401 (2015).

17. R. Re et al., "Probe-hosted silicon photomultipliers for time-domain functional near-infrared spectroscopy: phantom and in vivo tests," Neurophotonics 3(4), 045004 (2016).

18. C. Veerappan et al., "A $160 \times 128$ single-photon image sensor with on-pixel 55 ps 10 b time-to-digital converter," in IEEE Int. SolidState Circuits Conf. Digest of Technical Papers (ISSCC 2011), pp. 312-314 (2011).

19. J. Nissinen and J. Kostamovaara, "A high repetition rate CMOS driver for high-energy sub-NS laser pulse generation in SPAD-based time-offlight range finding," IEEE Sens. J. 16(6), 1628-1633 (2016).

20. B. Ryvkin et al., "Asymmetric-waveguide laser diode for high-power optical pulse generation by gain switching," J. Lightwave Technol. 27(12), 2125-2131 (2009).

21. B. S. Ryvkin et al., "Quantum well laser with an extremely large active layer width to optical confinement factor ratio for high-energy single picosecond pulse generation by gain switching," Semicond. Sci. Technol. 26(4), 045010 (2011).

22. B. S. Ryvkin et al., "Vertical cavity surface emitting lasers with the active layer position detuned from standing wave antinode for picosecond pulse generation by gain switching," J. Appl. Phys. 110(12), 123101 (2011).

23. B. Lanz et al., "Performance improvement by a saturable absorber in gain-switched asymmetric-waveguide laser diodes," Opt. Express 21(24), 29780-29791 (2013).

24. H. Wabnitz et al., "Performance assessment of time-domain optical brain imagers, part 1: basic instrumental performance protocol," J. Biomed. Opt. 19(8), 086010 (2014).

25. S. Konugolu Venkata Sekar et al., "Broadband $(600-1350 \mathrm{~nm})$ timeresolved diffuse optical spectrometer for clinical use," IEEE J. Sel. Top. Quantum Electron. 22(3), 7100609 (2016).

26. D. Contini et al., "Multi-channel time-resolved system for functional near infrared spectroscopy," Opt. Express 14(12), 5418-5432 (2006).

27. E. Martinenghi et al., "Time-resolved single-photon detection module based on silicon photomultiplier: a novel building block for time-correlated measurement systems," Rev. Sci. Instrum. 87(7), 073101 (2016).

28. A. Pifferi et al., "Performance assessment of photon migration instruments: the MEDPHOT protocol," Appl. Opt. 44(11), 2104-2114 (2005).

29. D. Contini et al., "Photon migration through a turbid slab described by a model based on diffusion approximation. I. Theory," Appl. Opt. 36(19), 4587-4599 (1997).

30. H. Wabnitz et al., "Performance assessment of time-domain optical brain imagers, part 2: nEUROPt protocol," J. Biomed. Opt. 19(8), 086012 (2014).

31. A. Pifferi et al., "Mechanically switchable solid inhomogeneous phantom for performance tests in diffuse imaging and spectroscopy," J. Biomed. Opt. 20(12), 121304 (2015).

32. D. Contini et al., "Novel method for depth-resolved brain functional imaging by time-domain NIRS," Proc. SPIE 6629, 662908 (2007).

Laura Di Sieno received her master's degree in electronics engineering at the Politecnico di Milano, Italy, in 2011, and her PhD in physics at the same university in 2015. Since January 2015, she has been a postdoctoral fellow. Her activity is mainly focused on the study and application of instrumentation for time-resolved optical spectroscopy of highly scattering media.

Jan Nissinen received his MSc Engineering, Licentiate of Tech. and Dr. Tech. degrees in electrical engineering from the University of Oulu, Finland, in 2002, 2007, and 2011, respectively. He has been a research scientist from 2002 to 2011 and postdoctoral researcher since 2011 in Electronics Laboratory at the University of Oulu. His research interests include the design of mixed-signal integrated circuits for pulsed time-of-flight laser rangefinders and for pulsed Raman spectroscopy.

Lauri Hallman received his MSc and Dr. Tech. degrees in electrical engineering from the University of Oulu, Oulu, Finland, in 2006 and 
2015 , respectively. He has done research in laser transmitter development and pulsed time-of-flight range finding. Since 2016, he has been a postdoctoral researcher at the Circuits and Systems Research Unit, University of Oulu.

Edoardo Martinenghi received his master's degree in electronics engineering from Politecnico di Milano in 2013 and his PhD in physics in 2016. Currently, he is working as a postdoc researcher. His research interests include design of photodetectors for time-domain diffuse optics and its application to biological media.

Davide Contini received his master's degree in electronics engineering and his $\mathrm{PhD}$ in physics from the Politecnico di Milano, Milan, Italy, in 2003 and 2007, respectively. Since 2014, he has been an associate professor in physics at the Department of Physics, Politecnico di Milano. His research activity is mainly focused on the study of time-resolved spectroscopy of highly diffusive media for applications in biology and medicine.

Antonio Pifferi is a full professor in the Department of Physics, Politecnico di Milano, Italy. He received his MS degree in nuclear engineering from Politecnico di Milano in 1991 and his PhD in physics from Politecnico di Torino in 1995. He was a visiting scientist at Lund
Institute of Technology, Sweden, from 1995 to 1996. His research focuses on the development of new laser techniques and instrumentation for diagnosis, and on the study of light propagation in diffusive media, with applications to optical biopsy, optical mammography, and functional brain imaging.

Juha Kostamovaara received his degrees of Dipl. Eng. and Dr. Tech in electrical engineering from the University of Oulu, Finland, in 1980 and 1987, respectively. In 1995, he was invited to become full professor of electronics at the University of Oulu, where he is currently also the head of the Circuits and Systems Research Unit. His main interests are in the development of high-speed electronic circuits and systems and their applications in electronic and optoelectronic measurements.

Alberto Dalla Mora is an assistant professor of physics at Politecnico di Milano, Italy, since 2011. From Politecnico di Milano, he received cum laude his master's degree in electronics engineering and his $\mathrm{PhD}$ in information and communication technology in 2006 and 2010 respectively. He authored more than 40 papers in international peer-reviewed journals and more than 40 conference proceedings. His research interests include time-resolved diffuse optics techniques and instrumentation for biomedical applications. 